\title{
Comprimento e largura do tamanho ótimo da parcela experimental em batata
}

\author{
Length and width of the optimal experimental plot size in potato
}

\author{
Lindolfo Storck $^{1}$ Sérgio José Ribeiro de Oliveira ${ }^{2}$ \\ Danton Camacho Garcia ${ }^{3}$ Dílson Antônio Bisognin ${ }^{3}$
}

\section{RESUMO}

A redução do erro experimental e o conseqüente aumento da precisão experimental podem ser obtidos com a aplicação do tamanho ótimo de parcelas experimentais. $O$ objetivo deste trabalho foi investigar as relações da largura e do comprimento sobre a estimativa do tamanho ótimo de parcela na cultura de batata pelo método da regressão múltipla. Doze ensaios de uniformidade de batata da cultivar Macaca foram conduzidos na estação experimental da Fundação Estadual de Pesquisa Agropecuária em Júlio de Castilhos, Rio Grande do Sul. Cada ensaio foi constituído por 12 linhas com 24 covas. Foram estimados o ponto crítico e a natureza da função de superfície de resposta do coeficiente de variação em relação às diferentes larguras e comprimentos das parcelas planejadas. A análise de causa e efeito foi feita usando-se o tamanho ótimo de parcela como variável dependente e as estimativas dos parâmetros da função de superfície de resposta como variáveis independentes. O tamanho ótimo de parcela pode ser estimado procedendo-se a colheita das covas em linhas independentes. O efeito linear do comprimento é quem define o tamanho ótimo de parcela na cultura da batata. O tamanho ótimo de uma parcela experimental de batata é de 24 covas distribuídas em uma linha.

Palavras-chave: Solanum tuberosum L., tamanho de parcela, precisão experimental, superfície de resposta, análise de trilha.

\section{ABSTRACT}

The use of optimal plot size can reduce random error and increase experimental precision. The objective was to identify

\begin{abstract}
the best relationship between length and width and optimal experimental plot size in potato with the multiple regression method. Twelve homogeny trials of 12 rows and 24 hills of the potato cultivar Macaca were carried out at the experimental area of the Fundação Estadual de Pesquisa Agropecuária, Júlio de Castilhos, Rio Grande do Sul State. The multiple regression critical point and function type were estimated for the variation coefficient of each designed plot length and width. Cause and effect analysis was done considering optimal plot size as dependent and estimation parameters of the multiple regression function as independent variables. Hill production of independent rows can be used to estimate optimal plot size. The optimal plot size in potato depends upon the linear effect of plot length. The optimal experimental plot size in potato is one row with 24 hills.
\end{abstract}

Key words: Solanum tuberosum L., experimental precision, response surfaces, path analysis.

\section{INTRODUÇÃO}

O erro experimental, o qual consiste na variância existente entre unidades experimentais que receberam o mesmo tratamento, é estimado mediante a aplicação da repetição, que é um dos princípios da experimentação. Várias fontes são responsáveis pelo erro experimental, como a heterogeneidade do material experimental, as competições intra e interparcelar e a heterogeneidade do solo. Esta última é decorrente de várias causas, tais como diferenças

${ }^{1}$ Departamento de Fitotecnia, Universidade Federal de Santa Maria (UFSM), 97105-900 Santa Maria, RS, Brasil. E-mail: lindolfo@smail.ufsm.br Autor para correspondência.

${ }^{2}$ Departamento de Ciências Exatas e Tecnológicas, Universidade Estadual de Santa Cruz, 45650-000, Ilhéus, BA, Brasil.

${ }^{3}$ Departamento de Fitotecnia, UFSM, 97105-900 Santa Maria, RS, Brasil. 
entre fertilidade, drenagem, relevo, manejo ou resíduos de culturas anteriores, aplicação de fertilizantes para a implantação do experimento (GOMEZ \& GOMEZ, 1984; STEEL et al., 1997; STORCK et al., 2000) e diferenças climáticas e edáficos mais intensificadas em regiões de clima tropical (RAMALHO et al., 2000).

A redução do erro experimental e o conseqüente aumento da precisão experimental podem ser propostos a partir da aplicação de métodos de determinação do tamanho ótimo de parcelas experimentais. Existem diversos métodos que, aplicados a um mesmo conjunto de dados, proporcionam os mais variados resultados. O método gráfico ou da máxima curvatura relaciona, num sistema de coordenadas, os tamanhos de parcela com as variâncias, ou coeficientes de variação, formando uma curva, sendo o tamanho ótimo indicado pela maior distância entre a curva e uma reta que liga as extremidades (FEDERER, 1955). Outro método relaciona a variância de parcelas com o índice de heterogeneidade do solo (SMITH, 1938). Existem ainda os métodos algébricos, que estimam o tamanho ótimo de parcelas usando as estimativas dos parâmetros de funções apropriadas que relacionam as variâncias com os tamanhos de parcelas planejados (LESSMAN \& ATKINS, 1963; MEIER \& LESSMAN, 1971; THOMAS, 1974). Esses métodos indicam o tamanho de parcela considerado ótimo sem fornecer informações sobre a forma mais adequada. O método da regressão múltipla (LUGO, 1977) baseia-se no modelo de superfície de resposta, no qual o coeficiente de variação dos diversos tamanhos e formas de parcela é a variável dependente (Y). A função

$$
Y=\beta_{0}+\beta_{1} X_{1}+\beta_{11} X_{1}^{2}+\beta_{2} X_{2}+\beta_{22} X_{2}^{2}+\beta_{12} X_{1} X_{2}+\varepsilon
$$

onde $\mathrm{X}_{1}$ é o número de linhas (largura) e $\mathrm{X}_{2} \mathrm{o}$ número de colunas (comprimento) da parcela experimental, tal que $\mathrm{X}=\mathrm{X}_{1} * \mathrm{X}_{2}$ é o tamanho da parcela em número de unidades básicas. $\mathrm{O}$ ponto crítico da função, igualando as derivadas parciais em relação a $X_{1}$ e $X_{2}$ a menos um, indica o tamanho e a forma ótimos de parcela. A natureza e a coerência desse ponto (tanto de máximo, mínimo ou cela) são estudadas pelos autovalores da matriz das variáveis independentes (DRAPER \& SMITH, 1981). Apesar de haver várias aplicações do método de regressão múltipla (LUGO, 1977; STORCK \& UITDEWILLIGEN, 1980; OLIVEIRA, 1994; ZANON, 1996), não há estudos a respeito dos efeitos diretos e indiretos das dimensões da largura e do comprimento sobre a área do tamanho ótimo determinado pelo método.

O objetivo deste trabalho foi investigar as relações da largura e do comprimento sobre a estimativa do tamanho ótimo de parcela pelo método da superfície de resposta na cultura de batata.

\section{MATERIAL E MÉTODOS}

Doze ensaios de uniformidade com a cultivar macaca de batata foram conduzidos na estação experimental da Fundação Estadual de Pesquisa Agropecuária (FEPAGRO) em Júlio de Castilhos, Rio Grande do Sul. O solo é um latossolo vermelho escuro distrófico. O manejo da cultura seguiu recomendações técnicas para o cultivo da batata (BISOGNIN, 1997). A colheita e a determinação do rendimento de tubérculos de cada cova foram feitas nos dias $28 \mathrm{de}$ novembro e primeiro de dezembro de 2003. Cada ensaio foi constituído por 12 linhas de 24 covas, espaçadas $25 \mathrm{~cm}$ na linha e $80 \mathrm{~cm}$ entre linhas. Cada cova foi definida como sendo uma unidade básica (UB) e identificada pelo número de ordem da linha e coluna. Planejaram-se, para cada um dos 12 ensaios, 18 tipos distintos de parcelas, com diferentes números de linhas (L) e colunas $(\mathrm{C})(\mathrm{L} \leq \mathrm{C})$, com os respectivos números de repetições (Tabela 1 ).

Para cada tipo de parcela com $\mathrm{X}$ unidades básicas de tamanho, identificada pelo número de linhas $\left(\mathrm{X}_{1}\right)$ e de colunas $\left(\mathrm{X}_{2}\right)$ ou comprimento da linha, tal que $\mathrm{X}=\mathrm{X}_{1}{ }^{*} \mathrm{X}_{2}$, foi estimado o coeficiente de variação, $\mathrm{CV}(\mathrm{x})$. Com os dados ajustou-se o modelo de superfície de resposta, método da regressão múltipla (LUGO, 1977), $Y=\beta_{0}+\beta_{1} X_{1}+\beta_{11} X_{1}^{2}+\beta_{2} X_{2}+\beta_{22} X_{2}^{2}+\beta_{12} X_{1} X_{2}+\varepsilon$, em que a variável dependente (Y) é o CV(x), $\beta_{1}$ é o efeito da largura da parcela $\left(\mathrm{X}_{1}\right), \beta_{11}$ é o efeito quadrático da largura, $\beta_{2}$ é o efeito do comprimento da parcela $\left(\mathrm{X}_{2}\right)$, $\beta_{22}$ é o efeito quadrático do comprimento e $\beta_{12}$ é o

Tabela 1 - Número de repetições para as parcelas planejadas com diferentes comprimentos e larguras, em número de unidades básicas (covas) de batata.

\begin{tabular}{lcccc}
\hline \multirow{2}{*}{ Comprimento (C) } & \multicolumn{4}{c}{ Larguras (L) } \\
\cline { 2 - 5 } & 1 & 2 & 3 & 4 \\
\hline 1 & 288 & - & - & - \\
2 & 144 & 72 & - & - \\
3 & 96 & 48 & 32 & - \\
4 & 72 & 36 & 24 & 18 \\
6 & 48 & 24 & 16 & 12 \\
8 & 36 & 18 & 12 & 9 \\
\hline
\end{tabular}


efeito da interação linear entre largura e comprimento $\left(\mathrm{X}_{12}=\mathrm{X}_{1} * \mathrm{X}_{2}\right)$. Derivando a função em relação a $\mathrm{X}_{1}$ e $\mathrm{X}_{2}$ e igualando as derivadas parciais a menos um, obtémse o ponto crítico $\mathrm{X}^{+}=\left[\mathrm{X}_{1}^{+} \mathrm{X}_{2}^{+}\right]$da função, que é considerado o tamanho ótimo de parcela em número de covas de largura $\left(\mathrm{X}_{1}\right)$ e de comprimento $\left(\mathrm{X}_{2}\right)$ (LUGO, 1977), isto é:

$$
\begin{aligned}
& 2 \hat{\beta}_{11} X_{1}+\hat{\beta}_{12} X_{2}=-1-\hat{\beta}_{1} \\
& \hat{\beta}_{12} X_{1}+2 \hat{\beta}_{22} X_{2}=-1-\hat{\beta}_{2}
\end{aligned}
$$

Usando a teoria matricial (DRAPER \& SMITH, 1981) pode-se escrever o sistema na forma $\mathrm{AX}=\mathrm{K}$, ou seja:

$$
\left[\begin{array}{ll}
2 \hat{\beta}_{11} & \hat{\beta}_{12} \\
\hat{\beta}_{12} & 2 \hat{\beta}_{22}
\end{array}\right] *\left[\begin{array}{l}
X_{1} \\
X_{2}
\end{array}\right]=\left[\begin{array}{ll}
-1 & \hat{\beta}_{1} \\
-1 & \hat{\beta}_{2}
\end{array}\right]
$$

em que a solução é $\mathrm{X}^{+}=\mathrm{A}^{-1} \mathrm{~K}$. O ponto crítico $\mathrm{X}^{+}$é o vetor com $\mathrm{X}_{1}^{+}$(número de $\mathrm{UB}^{\prime} \mathrm{s}$ de linhas) e $\mathrm{X}_{2}^{+}$ (número de UB's de comprimentos). Procedendo $\mathrm{X}_{12}{ }^{+}=\left(\mathrm{X}_{1}^{+}\right)^{*}\left(\mathrm{X}_{2}^{+}\right)$temos o tamanho e a forma da parcela. A natureza do ponto crítico (máximo, mínimo ou cela) é encontrada examinando-se os autovalores da matriz A (DRAPER \& SMITH, 1981).

Foram estimadas as correlações lineares entre a variável $\mathrm{X}_{12}{ }^{+} \mathrm{e}$ as estimativas dos efeitos do modelo de superfície de resposta $\left(\hat{\beta}_{0}, \hat{\beta}_{1}, \hat{\beta}_{11}, \hat{\beta}_{2}, \hat{\beta}_{22}\right.$, $\hat{\beta}_{12}$ ). Na matriz das correlações, utilizou-se o teste de multicolinearidade (CRUZ, 2001; CRUZ \& CARNEIRO, 2003). Após a eliminação das variáveis causadoras de multicolinearidade, procedeu-se a análise de trilha ou de causa e efeito (VENCOVSKY \& BARRIGA, 1992; CRUZ \& REGAZZI, 1994) usando $\mathrm{X}_{12}{ }^{+}$como variável principal.

Para os cálculos estatísticos, foi adotado um erro de conclusão igual a $5 \%$ e utilizados os seguintes pacotes estatísticos: software científico NTIA, desenvolvido pelo Centro Tecnológico para Informática (EMBRAPA, 1997), o programa GENES (CRUZ, 2001), o programa SAEG (RIBEIRO JÚNIOR, 2001) e um programa escrito em linguagem Fortram IV (ABOU-EL-FITTOUH et al., 1974), com algumas modificações, específico para calcular a média e a variância de parcelas de diferentes tamanhos.

\section{RESULTADOS E DISCUSSÃO}

Os coeficientes de variação (CV) decresceram com o aumento do tamanho das parcelas, para cada um dos doze ensaios de 288 unidades básicas, de acordo com a lei empírica de SMITH
(1938). Além disso, ocorreu uma variação de 25,3\% a $45,2 \%$ para o $\mathrm{CV}(\mathrm{x}=1)$ e entre $2,5 \%$ e $16,3 \%$ para $\mathrm{CV}(\mathrm{x}=4 * 8=32)$ nos doze ensaios (Tabela 2$)$. Verificase, a partir desses percentuais, que o conjunto de ensaios foi adequado para o estudo proposto, pois o comportamento do $\mathrm{CV}$ em relação ao tamanho das parcelas foi variável tal como encontrado por outros autores (MEIER \& LESSMAN, 1971; LUGO, 1977; STORCK \& UITDEWILLIGEN, 1980; ZANON, 1996).

Para todos os ensaios, a qualidade do ajuste da função de superfície de resposta foi adequada, pois o coeficiente de determinação foi alto (média de 0,9527 ) e pouco variável (desvio padrão igual a 0,03 ) (Tabela 3). Também as estimativas dos efeitos lineares e quadráticos do comprimento e a interação linear entre largura e comprimento foram as mais consistentes (baixo desvio padrão). Já os efeitos linear e quadrático da largura e a constante foram os mais variáveis (alto desvio padrão), podendo explicar a falta de significância, na metade dos ensaios avaliados, do efeito quadrático da largura $\left(\hat{\beta}_{11}\right)$.

Na cultura da batata, a variação do tamanho da parcela, quanto ao comprimento, independe da variação quanto à largura, pois o efeito da interação linear entre estas dimensões $\left(\hat{\beta}_{12}\right)$ não foi significativo nos doze ensaios. Assim, é possível estimar o tamanho ótimo de parcela usando apenas os comprimentos das linhas, já que a variação entre o número de linhas não foi significativa em alguns casos. Outra conseqüência da independência da variação das linhas e das colunas é a possibilidade de se dispensar o uso de bordaduras nos experimentos. Esses resultados são de grande aplicação prática, já que os experimentos com batata têm custo elevado de implantação e manejo da cultura, pois requerem muitos cuidados para a aplicação dos tratos culturais e a utilização de tubérculos-sementes de diferentes idades fisiológicas. Este fato pode resultar em altos coeficientes de variação, o que limita o formato e o tamanho das parcelas experimentais.

Apenas em um dos doze ensaios foi observado o ponto crítico de cela, máximo para largura e mínimo para o comprimento (Tabela 3). Resultados com dimensões negativas também foram encontrados por outros pesquisadores (STORCK \& UITDERWILLIGEN, 1980; STORCK et al., 1982 e ZANON, 1996), que desaconselham a utilização de tal método para o dimensionamento de parcelas experimentais.

No presente trabalho, o ensaio seis foi excluído dos procedimentos de estudo de causa e efeito para evitar distorções dos resultados, já que se tratou de um único caso em doze repetições. Nos demais onze 
Tabela 2 - Coeficientes de variação (\%) entre parcelas com diferentes tamanhos medidos em número de unidades básicas de larguras (L) e comprimentos $(\mathrm{C})$ e número de repetições $(\mathrm{N})$ em doze ensaios.

\begin{tabular}{|c|c|c|c|c|c|c|c|c|c|c|c|c|c|c|}
\hline \multirow{2}{*}{$\mathrm{L}$} & \multirow{2}{*}{$\mathrm{C}$} & \multirow{2}{*}{$\mathrm{N}$} & \multicolumn{12}{|c|}{ Ensaio } \\
\hline & & & 1 & 2 & 3 & 4 & 5 & 6 & 7 & 8 & 9 & 10 & 11 & 12 \\
\hline 1 & 1 & 288 & 33,0 & 26,8 & 33,2 & 28,4 & 43,5 & 25,3 & 45,2 & 30,0 & 39,8 & 32,6 & 34,0 & 39,9 \\
\hline 1 & 2 & 144 & 23,5 & 19,8 & 25,1 & 19,7 & 35,0 & 18,6 & 36,9 & 23,3 & 32,4 & 26,3 & 28,7 & 33,1 \\
\hline 1 & 3 & 96 & 20,8 & 16,8 & 22,0 & 17,8 & 32,8 & 17,6 & 32,9 & 22,3 & 29,2 & 24,6 & 27,9 & 30,4 \\
\hline 1 & 4 & 72 & 19,5 & 16,1 & 20,7 & 15,8 & 29,8 & 15,9 & 30,3 & 19,9 & 27,8 & 23,9 & 26,6 & 29,0 \\
\hline 1 & 6 & 48 & 17,8 & 13,8 & 18,9 & 14,4 & 28,5 & 14,2 & 26,9 & 19,3 & 26,5 & 22,5 & 26,2 & 27,7 \\
\hline 1 & 8 & 36 & 17,2 & 13,5 & 17,1 & 12,7 & 27,6 & 13,4 & 26,7 & 17,7 & 26,0 & 21,8 & 25,4 & 26,8 \\
\hline 2 & 2 & 72 & 17,3 & 14,6 & 19,0 & 14,8 & 23,3 & 15,1 & 25,4 & 19,1 & 20,7 & 19,4 & 18,8 & 25,3 \\
\hline 2 & 3 & 48 & 15,2 & 13,1 & 15,9 & 13,5 & 22,4 & 14,3 & 23,0 & 19,1 & 17,8 & 18,9 & 17,8 & 22,5 \\
\hline 2 & 4 & 36 & 14,4 & 12,7 & 14,8 & 12,1 & 19,9 & 13,6 & 21,0 & 17,8 & 16,7 & 18,2 & 16,8 & 22,2 \\
\hline 2 & 6 & 24 & 13,5 & 11,4 & 13,8 & 11,1 & 19,2 & 12,5 & 19,2 & 17,0 & 15,1 & 18,0 & 16,7 & 21,3 \\
\hline 2 & 8 & 18 & 13,7 & 11,0 & 11,9 & 10,2 & 18,8 & 11,5 & 18,2 & 16,2 & 15,0 & 17,7 & 15,9 & 20,8 \\
\hline 3 & 3 & 32 & 13,9 & 11,2 & 13,8 & 12,0 & 20,7 & 9,3 & 18,8 & 15,1 & 9,8 & 17,9 & 10,6 & 17,0 \\
\hline 3 & 4 & 24 & 12,9 & 10,9 & 12,7 & 10,8 & 18,7 & 8,4 & 16,5 & 14,0 & 9,2 & 17,8 & 9,5 & 16,2 \\
\hline 3 & 6 & 16 & 12,8 & 9,9 & 12,4 & 10,1 & 18,0 & 7,7 & 14,5 & 13,2 & 7,9 & 17,2 & 9,0 & 15,4 \\
\hline 3 & 8 & 12 & 12,2 & 10,2 & 10,9 & 9,0 & 17,9 & 7,2 & 12,6 & 12,4 & 6,4 & 17,8 & 7,6 & 14,7 \\
\hline 4 & 4 & 18 & 8,0 & 8,0 & 11,2 & 7,0 & 17,0 & 6,0 & 11,8 & 7,3 & 12,3 & 7,2 & 12,8 & 11,1 \\
\hline 4 & 6 & 12 & 8,0 & 6,7 & 11,3 & 6,2 & 16,2 & 5,0 & 11,9 & 7,1 & 11,1 & 7,3 & 13,3 & 10,2 \\
\hline 4 & 8 & 9 & 7,8 & 7,0 & 9,6 & 4,7 & 16,3 & 2,5 & 9,5 & 5,3 & 10,9 & 7,0 & 12,3 & 9,3 \\
\hline
\end{tabular}

Tabela 3 - Estimativas dos parâmetros da função $Y=\beta_{0}+\beta_{1} X_{1}+\beta_{11} X_{1}^{2}+\beta_{2} X_{2}+\beta_{22} X_{2}^{2}+\beta_{12} X_{1} X_{2}+\varepsilon$, em que $Y$ é o coeficiente de variação, coeficiente de determinação $\left(\mathrm{R}^{2}\right)$ e ponto crítico identificado por $\mathrm{X}_{1}^{+}$larguras e $\mathrm{X}_{2}^{+}$comprimentos e $\mathrm{X}_{12}{ }^{+}=\mathrm{X}_{1}^{+} \mathrm{X}_{2}^{+}$para o tamanho ótimo de parcela em número de covas e natureza do ponto crítico obtido em doze ensaios.

\begin{tabular}{lccccccccccc}
\hline Ensaio & $\beta_{0}$ & $\beta_{1}$ & $\beta_{11}$ & $\beta_{2}$ & $\beta_{22}$ & $\beta_{12}$ & $\mathrm{R}^{2}$ & $\mathrm{X}_{1}^{+}$ & $\mathrm{X}_{2}^{+}$ & $\mathrm{X}_{12}{ }^{+}$ & Natureza $^{\text {na }}$ \\
\hline 1 & $40,0080^{*}$ & $-6,6273^{*}$ & $0,3493^{\text {ns }}$ & $-5,3632^{*}$ & $0,3788^{*}$ & $0,2913^{\text {ns }}$ & 0,9228 & 7,7811 & 4,0878 & 31,8078 & Mínimo \\
2 & $32,8363^{*}$ & $-5,2569^{*}$ & $0,292^{\text {ns }}$ & $-4,4628^{*}$ & $0,3045^{*}$ & $0,2532^{\text {ns }}$ & 0,9410 & 7,0849 & 4,3824 & 31,0490 & Mínimo \\
3 & $43,7498^{*}$ & $-10,8280^{*}$ & $1,2897^{*}$ & $-4,7642^{*}$ & $0,2915^{*}$ & $0,3023^{\text {ns }}$ & 0,9520 & 3,4498 & 6,3839 & 22,0228 & Mínimo \\
4 & $33,4711^{*}$ & $-4,9306^{*}$ & $0,1955^{\text {ns }}$ & $-4,5192^{*}$ & $0,2910^{*}$ & $0,2271^{\text {ns }}$ & 0,9247 & 10,4761 & 3,6778 & 38,5295 & Mínimo \\
5 & $59,6822^{*}$ & $-16,9839^{*}$ & $2,3237^{*}$ & $-5,4564^{*}$ & $0,3770^{*}$ & $0,2711^{\text {ns }}$ & 0,9530 & 3,3016 & 6,0488 & 19,9709 & Mínimo \\
6 & $29,5682^{*}$ & $-3,8413^{*}$ & $-0,0753^{\text {ns }}$ & $-3,2631^{*}$ & $0,2092^{*}$ & $0,1152^{\text {ns }}$ & 0,9593 & $-16,1410$ & 12,2422 & $-197,602$ & Cela \\
7 & $61,0285^{*}$ & $-15,0427^{*}$ & $1,6233^{*}$ & $-6,0227^{*}$ & $0,3940^{*}$ & $0,2825^{\text {ns }}$ & 0,9807 & 4,1812 & 6,4312 & 26,8899 & Mínimo \\
8 & $31,9071^{*}$ & $-0,9896^{\text {ns }}$ & $-0,8489^{*}$ & $-3,3120^{*}$ & $0,2032^{*}$ & $0,1842^{\text {ns }}$ & 0,9639 & 0,2871 & 8,0189 & 2,3021 & Mínimo \\
9 & $63,1548^{*}$ & $-24,8927^{*}$ & $3,7581^{*}$ & $-4,9106^{*}$ & $0,3547^{*}$ & $0,1222^{\text {ns }}$ & 0,9818 & 3,2084 & 6,3697 & 20,4362 & Mínimo \\
10 & $34,9908^{*}$ & $-1,7340^{\text {ns }}$ & $-0,8851^{\text {ns }}$ & $-3,3884^{*}$ & $0,2258^{\text {ns }}$ & $0,2513^{\text {ns }}$ & 0,9024 & 0,0792 & 7,4587 & 0,5905 & Mínimo \\
11 & $53,3557^{*}$ & $-22,1210^{*}$ & $3,3573^{*}$ & $-2,5712^{*}$ & $0,1724^{\text {ns }}$ & $0,0971^{\text {ns }}$ & 0,9734 & 3,1996 & 6,5557 & 20,9760 & Mínimo \\
12 & $50,3012^{*}$ & $-9,7598^{*}$ & $0,5869^{\text {ns }}$ & $-4,2921^{*}$ & $0,3002^{*}$ & $0,1568^{\text {ns }}$ & 0,9845 & 7,6257 & 5,1581 & 39,3339 & Mínimo \\
${ }^{\#}$ Média & 45,8623 & $-10,8333$ & 1,0948 & $-4,4603$ & 0,2994 & 0,2217 & 0,9527 & 4,6068 & 5,8703 & 23,0826 & \\
${ }^{\#}$ DP & 12,13 & 8,03 & 1,55 & 1,03 & 0,07 & 0,07 & 0,03 & 3,26 & 1,38 & 12,72 & \\
\hline
\end{tabular}

\# Valores estimados com a exclusão do ensaio seis (caso atípico). DP = Desvio padrão.

* Significativo pelo teste t em nível de $5 \%$ de probabilidade de erro; ${ }^{\text {ns }}$ não-significativo.

ensaios, o ponto crítico foi de mínimo, ou seja, a combinação das dimensões $\mathrm{X}_{1}^{+}$e $\mathrm{X}_{2}^{+}$determinou o menor coeficiente de variação da função (Tabela 3 ). Também, em dois casos (ensaios oito e dez), o valor crítico de $\mathrm{X}_{1}^{+}$foi menor que a unidade, talvez devido à não-significância do efeito linear da largura. Em outro caso, o número de linhas foi maior do que 10 (ensaio quatro), não permitindo repetições de linhas na área disponível. De qualquer forma, a variação de $\mathrm{X}_{1}^{+}$foi elevada $(\mathrm{DP}=3,26)$ em comparação à média (4,61 UB's). Os valores do tamanho ótimo de parcela oscilaram entre uma e 40 covas, sendo que a média

Ciência Rural, v. 35, n. 5, set-out, 2005. 
nos onze ensaios foi de 23 covas, próximo das 21 covas, distribuídas em uma linha, que se usa na experimentação de competição de cultivares de batata (ZVOMUYA et al., 2002; MILLER et al., 2004). Variações do tamanho ótimo de parcelas de batata também foram encontradas usando outras metodologias (OLIVEIRA, 1994). No entanto, para os ensaios de determinação de valor de cultivo e uso da batata, para fins de registro no Ministério da Agricultura do Brasil, são requeridas, no mínimo, 100 covas distribuídas em quatro linhas úteis (SNPC, 2003).

Houve uma associação negativa e significativa entre as estimativas dos parâmetros que representam o efeito linear e quadrático da largura $\left(\hat{\beta}_{1}\right.$ e $\hat{\beta}_{11}$ ), assim como os que dizem respeito ao efeito linear e quadrático do comprimento $\left(\hat{\beta}_{2}\right.$ e $\left.\hat{\beta}_{22}\right)$ (Tabela 4). Quanto maior for a magnitude do efeito linear da largura (linhas) e/ou do efeito linear do comprimento, menor será o efeito quadrático sobre as respectivas dimensões nos onze ensaios. Por isto, constatou-se colinearidade severa (CRUZ, 2001; CRUZ \& CARNEIRO, 2003), a qual só foi reduzida para colinearidade fraca através da eliminação das variáveis $\hat{\beta}_{0}, \hat{\beta}_{11}$ e $\hat{\beta}_{22}$, o que permite estimativas não tendenciosas dos efeitos diretos e indiretos.

A análise de causa e efeito consistiu das estimativas dos parâmetros $\hat{\beta}_{1}, \hat{\beta}_{2}$ e $\hat{\beta}_{12}$ em função do tamanho ótimo de parcela (Tabela 5), sendo que a maior correlação foi entre o tamanho ótimo com $\hat{\beta}_{2}$ (efeito linear do comprimento). Isto indica que, quanto maior for o ponto crítico (área da parcela) mais

Tabela 4 - Correlação linear simples entre as estimativas dos parâmetros da função $Y=\beta_{0}+\beta_{1} X_{1}+\beta_{11} X_{1}^{2}+\beta_{2} X_{2}+\beta_{22} X_{2}^{2}+\beta_{12} X_{1} X_{2}$ e $\mathrm{o}$ tamanho ótimo de parcela $\left(\mathrm{X}_{12}{ }^{+}\right)$em onze ensaios.

\begin{tabular}{ccccccc}
\hline & $\hat{\beta}_{1}$ & $\hat{\beta}_{11}$ & $\hat{\beta}_{2}$ & $\hat{\beta}_{22}$ & $\hat{\beta}_{12}$ & $\mathrm{X}_{12}{ }^{+}$ \\
\hline$\hat{\beta}_{0}$ & $-0,9019^{*}$ & $0,8513^{*}$ & $-0,4109^{\mathrm{ns}}$ & $0,4711^{\mathrm{ns}}$ & $-0,2542^{\mathrm{ns}}$ & $0,1386^{\mathrm{ns}}$ \\
$\hat{\beta}_{1}$ & - & $-0,9915^{*}$ & $0,1787^{\mathrm{ns}}$ & $-0,2551^{\mathrm{ns}}$ & $0,4515^{\mathrm{ns}}$ & $-0,1511^{\mathrm{ns}}$ \\
$\hat{\beta}_{11}$ & - & - & $-0,1720^{\mathrm{ns}}$ & $0,2434^{\mathrm{ns}}$ & $-0,4297^{\mathrm{ns}}$ & $0,1821^{\mathrm{ns}}$ \\
$\hat{\beta}_{2}$ & - & - & - & $-0,9773^{*}$ & $-0,6076^{*}$ & $-0,4526^{\mathrm{ns}}$ \\
$\hat{\beta}_{22}$ & - & - & - & - & $0,4943^{\mathrm{ns}}$ & $0,4776^{\mathrm{ns}}$ \\
$\hat{\beta}_{12}$ & - & - & - & - & - & $0,0574^{\mathrm{ns}}$ \\
\hline
\end{tabular}

Valores estimados com a exclusão do ensaio seis (caso atípico).

* Significativo pelo teste $\mathrm{t}$ em nível de $5 \%$ de probabilidade de erro; ns=não-significativo
Tabela 5 - Efeitos diretos e indiretos das estimativas dos parâmetros da largura $\left(\hat{\beta}_{1}\right)$, comprimento $\left(\hat{\beta}_{2}\right)$ e da interação linear $\left(\hat{\beta}_{12}\right)$ sobre o tamanho ótimo de parcela $\left(\mathrm{X}_{12}{ }^{+}\right)$estimado como ponto crítico da superfície de resposta.

\begin{tabular}{lccc}
\hline Parâmetro & Efeito & Estimativa & Total \\
\hline$\hat{\beta}_{1}$ & Direto sobre $\mathrm{X}_{12}{ }^{+}$ & 0,2613 & \\
& Indireto via $\hat{\beta}_{2}$ & $-0,1519$ & \\
& Indireto via $\hat{\beta}_{12}$ & $-0,2605$ & $-0,1511$ \\
Total & & \\
$\hat{\beta}_{2}$ & Direto sobre $\mathrm{X}_{12}{ }^{+}$ & $-0,8498$ & \\
& Indireto via $\hat{\beta}_{1}$ & 0,0467 & \\
& Indireto via $\hat{\beta}_{12}$ & 0,3505 & $-0,4526$ \\
$\hat{\beta}_{12}$ & Total & & \\
& Direto sobre $\mathrm{X}_{12}{ }^{+}$ & $-0,5769$ & \\
& Indireto via $\hat{\beta}_{1}$ & 0,1179 & \\
& Indireto via $\hat{\beta}_{2}$ & 0,5164 & \\
& Total & & 0,0574 \\
\hline
\end{tabular}

negativo será o valor de $\hat{\beta}_{2}$, ou seja, maior será o efeito do comprimento sobre o tamanho da parcela.

$\mathrm{O}$ efeito direto de $\hat{\beta}_{2}$ sobre o tamanho ótimo de parcela $\left(\mathrm{X}_{12}^{+}\right)$tem sinal e magnitude semelhantes à correlação entre $\hat{\beta}_{2}$ e $X_{12}{ }^{+}$. Neste caso, conforme interpretação apresentada por VENCOVSKY \& BARRIGA (1992), $\hat{\beta}_{2}$ é quem explica a verdadeira associação entre $\hat{\beta}_{2}$ e $X_{12}{ }^{+}$, devendo-se ignorar as outras variáveis $\left(\hat{\beta}_{1}\right.$ e $\left.\hat{\beta}_{12}\right)$. Assim, a magnitude da influência da variação no comprimento das parcelas é o fator determinante do tamanho de parcela estimado como ponto crítico de uma função de superfície de resposta para o rendimento da cultura da batata. Por exclusão, a variação na largura (uso de duas ou mais linhas), bem como a interação linear entre largura e comprimento (não-significativos), têm suas influências reduzidas. Assim, o tamanho ótimo de parcela experimental na cultura de batata pode ser constituído por uma linha de 24 covas. Também, no aspecto da metodologia para estimar o tamanho ótimo de parcelas pode-se usar apenas o rendimento das covas de algumas linhas da área experimental, o que contempla grande redução de recursos humanos.

Ciência Rural, v.35 n. 5, set-out, 2005. 


\section{CONCLUSÕES}

O tamanho ótimo de parcela pode ser estimado procedendo-se à colheita das covas em linhas independentes; o efeito linear do comprimento da parcela (número de covas na linha) é quem define o tamanho ótimo de parcela na cultura da batata; e o tamanho ótimo de parcela é uma linha de 24 covas de comprimento.

\section{REFERÊNCIAS}

ABOU-EL-FITTOUH, H.A. et al. A program for studying the optimum plot size in field experiments on the computer. Agricultural Research Review, v.52, p.85$90,1974$.

BISOGNIN, D.A. (Ed.). Recomendações técnicas para o cultivo da batata no Rio Grande do Sul e Santa Catarina Santa Maria: Universidade Federal de Santa Maria, 1997. 64 p.

CRUZ, C.D. Programa GENES; versão windows; aplicativo computacional em genética e estatística Viçosa: UFV, 2001. 648p.

CRUZ, C.D.; CARNEIRO, P.C.S. Modelos biométricos aplicados ao melhoramento genético volume 2 . Viçosa: UFV, 2003. 585 p.

CRUZ, C.D.; REGAZZI, A.J. Modelos biométricos aplicados ao melhoramento genético. Viçosa: UFV, 1994. 390p.

DRAPER, N.R.; SMITH, H. Applied regression analysis. 2.ed. New York: John Wiley, 1981. 709p.

EMBRAPA. Ambiente de software NTIA, versão 4.2.2: manual do usuário - ferramental estatístico. Campinas: Centro Nacional de Pesquisa Tecnológica em Informática para a Agricultura, 1997. 258p.

FEDERER, W.T. Experimental design. New York: McMillan, 1955. 544p.

GOMEZ, K.A.; GOMEZ, A.A. Statistical procedures for agricultural research. 2.ed. New York: John Wiley, 1984. 680p.

LESSMAN, K.J.; ATKINS, R.E. Optimum plot size and relative efficiency of lattice designs for grain sorghum yield tests. Crop Science, v.3, n.6, p.477-481, 1963.

LUGO, F.C. Tamaño de parcela experimental y su forma. Revista Facultad Agronomia, v.9, n.3, p.55-74, 1977.

MEIER, V.D.; LESSMAN, K.J. Estimation of optimum field plot shape and size testing yield in Crambe abyssinica Hordnt. Crop Science, v.11, n.5, p.648-650, 1971.
MILLER, J.C. et al. Discriminating Russet Norkotah intraclonal selections Using canonical and cluster analysis. American Journal of Potato Research, v.81, n.3, p.203207, 2004

OLIVEIRA, P.H. Tamanho e forma ótimos da parcela para avaliação do rendimento em experimentos com batata. 1994. 83f. Dissertação (Mestrado em Agronomia) Programa de Pós-graduação em Agronomia, Universidade Federal de Santa Maria.

RAMALHO, M.A.P. et al. A experimentação em genética e melhoramento de plantas. Lavras: EDUFLA, 2000. 326p.

RIBEIRO JÚNIOR, J.I. Análises estatísticas no SAEG Viçosa: UFV, 2001. 301p.

SMITH, H.F. An empirical law describing heterogeneity in the yields of agricultural crops. Journal Agricultural Science, n.28, p.1-23, 1938 .

SNPC - Serviço Nacional de Proteção de Cultivares, Anexo III da Lei $N^{\circ} \mathbf{1 0 . 7 1 1}$, de 5 de agosto de 2003 (DOU de 06.08.2003), que dispõe sobre o Sistema Nacional de Sementes e Mudas. 2003.

STEEL, R.G.D. et al. Principles and procedures of statistics: a biometrical approach. 3.ed. New York: McGraw Hill Book, 1997. $666 \mathrm{p}$.

STORCK, L. et al. Comparação de métodos de estimativa do índice de heterogeneidade do solo e do tamanho ótimo de parcela em experimento com soja. Revista Centro de Ciências Rurais, v.2-3, n.12, p.189-202, 1982

STORCK, L. et al. Experimentação vegetal. Santa Maria: UFSM, 2000. 198p.

STORCK, L.; UITDEWILLIGEN, W.P.M. Estimativa para tamanho e forma de parcela e número de repetições para experimentos com milho (Zea mays L.). Agronomia Sulriograndense, v.12, n.2, p.269-282, 1980.

THOMAS, E.J. Relationship between plot size and plot variance. Agricultural Research Journal of Kerala, v.12, n.2, p.178-189, 1974.

VENCOVSKY, R.; BARRIGA, P. Genética biométrica no fitomelhoramento. Ribeirão Preto: Revista Brasileira de Genética, 1992. 496p.

ZANON, M.L.B. Tamanho e forma ótimos de parcelas experimentais para Eucaliptus saligna Smith. 1996. 78f. Dissertação (Mestrado em Engenharia Florestal), Programa de Pós-graduação em Engenharia Florestal, Universidade Federal de Santa Maria.

ZVOMUYA, F. et al. Response of Russet Norkotah intraclonal selections to nitrogen fertilization. American Journal of Potato Research, v.79, n.4, p.231-239, 2002. 